\title{
The Stability of Solutions for a Fractional Predator-Prey System
}

\author{
Yingjia Guo ${ }^{1,2}$ \\ ${ }^{1}$ School of Mathematics, Jilin University, Changchun 130012, China \\ ${ }^{2}$ Department of Mathematics, Jilin Agriculture Science and Technology College, Jilin 132101, China
}

Correspondence should be addressed to Yingjia Guo; guo.yingjia@163.com

Received 6 January 2014; Accepted 31 March 2014; Published 16 April 2014

Academic Editor: Changbum Chun

Copyright (C) 2014 Yingjia Guo. This is an open access article distributed under the Creative Commons Attribution License, which permits unrestricted use, distribution, and reproduction in any medium, provided the original work is properly cited.

We study a class of fractional predator-prey systems with Holling II functional response. A unique positive solution of this system is obtained. In order to prove the asymptotical stability of positive equilibrium for this system, we study the Lyapunov stability theory of a fractional system.

\section{Introduction}

We consider the following fractional predator-prey model with Holling Type II functional response:

$$
\begin{aligned}
& d^{\alpha} x(t)=x(t)\left(a-b x(t)-\frac{\gamma y(t)}{1+\beta x(t)}\right) d t^{\alpha}, \\
& d^{\alpha} y(t)=y(t)\left(-e+\frac{k \gamma x(t)}{1+\beta x(t)}\right) d t^{\alpha}, \quad(0<\alpha \leq 1),
\end{aligned}
$$

where $x(t)$ and $y(t)$ represent the population densities of prey and predator at time $t$, respectively. The parameters $a, b / a, \gamma$, $\beta, e$, and $k$ are positive constants that stand for prey intrinsic growth rate, carrying capacity, the maximum ingestion rate, half-saturation constant, predator death rate, and the conversion factor, respectively. One of the most popular predatorprey models with Holling Type II functional response is established in $[1,2]$. The asymptotic behavior of a stochastic predator-prey system with Holling Type II functional response is studied in [3]. The existence and asymptotical stability of equilibria and limit cycles for predator-prey systems with Holling II are obtained in [4]. A more complicated case about predator-prey systems is studied in $[5,6]$. White noise is always present in natural world; Liu et al. studied the asymptotic behavior of a stochastic predator-prey system with Holling II functional response.

However, in the real world, there are still many problems that cannot be solved by usual prey-predator model. Some complexity on multiscale analysis can be simplified by the fractional order calculus. Fractional differential equations have been studied in many other fields, for example, economic [7], physics [8-10], material [11], and so forth. In this paper, we present a fractional prey-predator model (1) to describe the ecosystem which performs well in the practical problem.

We will study long time behavior of system (1). If $a k \gamma>$ $a e \beta+b e$, then system (1) has a unique positive equilibrium:

$$
x^{*}=\frac{e}{k \gamma-e \beta}, \quad y^{*}=\frac{k \gamma(a k \gamma-a e \beta-b e)}{(k \gamma-e \beta)^{2}} .
$$

The positive equilibrium is asymptotically stable, which is proved in Section 5. The fractional derivative of (1) is modified Riemann-Liouville derivative, which is established in $[12,13]$. There are some good properties on the fractional derivative to study fractional system, such as the chain rule and fractional Taylor series. The details of modified RiemannLiouville derivative are given in Section 2. We show that there is a unique nonnegative solution of (1) in Section 3. In order to prove that the positive equilibrium is stable, we give a Lyapunov stability theorem of the fractional system in Section 4.

\section{Preliminaries}

2.1. Fractional Derivative via Fractional Difference. For an introduction to the classical fractional calculus we refer the reader to [14-17]. 
Definition 1. Let $f: \mathfrak{R} \rightarrow \mathfrak{R}, x \rightarrow f(x)$, be a continuous function, and let $h>0$ be a constant discretization span. Define the forward operator $F W(h)$; that is, (the symbol:= means that the left side is defined by the right one)

$$
F W(h) \cdot f(x):=f(x+h) .
$$

Then the fractional difference of order $\alpha, 0<\alpha<1$, of $f(x)$ is defined by the expression

$$
\begin{aligned}
\Delta^{\alpha} f(x) & :=(F W-1)^{\alpha} f(x) \\
& =\sum_{k=0}^{\infty}(-1)^{k}\left(\begin{array}{l}
\alpha \\
k
\end{array}\right) f(x+(\alpha-k) h),
\end{aligned}
$$

and its derivative of fractional order is defined by the expression

$$
f^{(\alpha)}(x)=\lim _{h \downarrow 0} \frac{\Delta^{\alpha} f(x)}{h^{\alpha}}, \quad 0<\alpha \leq 1 .
$$

2.2. Modified Riemann-Liouville Derivative. In this section we briefly review the main notions and results from the recent fractional calculus proposed by Jumarie $[12,18,19]$.

Definition 2 (Riemann-Liouville definition revisited). Refer to the function of Definition 1. Then its fractional derivative of order $\alpha$ is defined by the expression

$$
\begin{array}{r}
f^{(\alpha)}(x):=\frac{1}{\Gamma(-\alpha)} \int_{0}^{x}(x-\xi)^{-\alpha-1}(f(\xi)-f(0)) d \xi \\
\alpha<0 .
\end{array}
$$

For positive $\alpha$, one will set

$$
\begin{aligned}
f^{(\alpha)}(x) & =\left(f^{(\alpha-1)}(x)\right)^{\prime}, \quad 0<\alpha<1 \\
& =\frac{1}{\Gamma(1-\alpha)} \frac{d}{d x} \int_{0}^{x}(x-\xi)^{-\alpha}(f(\xi)-f(0)) d \xi \\
f^{(\alpha)}(x) & :=\left(f^{(n)}(x)\right)^{(\alpha-n)}, \quad n \leq \alpha<n+1, n \geq 1 .
\end{aligned}
$$

2.3. Useful Relations. Here we give some properties of the modified Riemann-Liouville derivative (see [13]) which are used further in this paper.

(i) Consider

$$
\Gamma(1+\alpha k)=:(\alpha k) !
$$

(ii) Useful differential relation:

$$
d^{\alpha} f \cong \Gamma(1+\alpha) d f, \quad 0<\alpha \leq 1 .
$$

(iii) Consider

$$
D^{\alpha} x^{\gamma}=\Gamma(1+\gamma) \Gamma^{-1}(\gamma+1-\alpha) x^{\gamma-\alpha}, \quad \gamma>0 .
$$

(iv) The chain rule:

$$
\begin{aligned}
(u(x) v(x))^{(\alpha)} & =u^{(\alpha)}(x) v(x)+u(x) v^{(\alpha)}(x), \\
(f[u(x)])^{(\alpha)} & =f_{u}^{\prime}(u) u^{(\alpha)}(x) .
\end{aligned}
$$

2.4. Integration with respect to $d t^{\alpha}$. The solution of the equation

$$
\begin{aligned}
& d^{\alpha} x=f(t) d t^{\alpha}, \quad t \geq 0, \\
& x(0)=x_{0}
\end{aligned}
$$

is defined by the following result (see [13]).

Lemma 3. Let $f(x)$ denote a continuous function; then the solution of (12) is defined by the equality

$$
\int_{0}^{t} f(\xi) d \xi^{\alpha}=\alpha \int_{0}^{t}(t-\xi)^{\alpha-1} f(\xi) d \xi, \quad 0<\xi \leq 1 .
$$

\section{Existence and Uniqueness of the Nonnegative Solution}

Theorem 4. For any initial value $(x(0), y(0)) \in R_{+}^{2}$, there is a unique global solution $(x(t), y(t))$ of system (1) on $t \geq 0$.

Proof. Note that the coefficients of system (1) are locally Lipschitz continuous for the given initial value $(x(0), y(0)) \in R^{2}$; there is a unique local solution $(x(t), y(t))$ on $t \in\left[0, \tau_{e}\right)$, where $\tau_{e}$ is the explosion time. Hence, we know that $(x(t)$, $y(t)), t \in\left[0, \tau_{e}\right)$ is a unique positive local solution of system (1). To show that this solution is global, we need to show that $\tau_{e}=\infty$. Let $m_{0} \geq 1$ be sufficiently large so that $x(0), y(0)$ all lie within the interval $\left[1 / m_{0}, m_{0}\right]$. For each integer $m \geq m_{0}$, define

$$
\begin{aligned}
\tau_{m}=\inf \{t & \in\left[0, \tau_{e}\right): \min \{x(t), y(t)\} \\
\leq & \left.\frac{1}{m} \text { or } \max \{x(t), y(t)\} \geq m\right\} .
\end{aligned}
$$

Clearly, $\tau_{m}$ is increasing as $m \rightarrow \infty$. Set $\tau_{\infty}=$ $\lim _{m \rightarrow \infty} \tau_{m}$, where $\tau_{\infty} \leq \tau_{e}$. If we can show that $\tau_{\infty}=\infty$, then $\tau_{e}=\infty$ and $(x(t), y(t)) \in R_{+}^{2}$ for all $t \geq 0$. In other words, to complete the proof all we need to show is that $\tau_{\infty}=\infty$. If this statement is false, then there is a constant $T>0$ such that

$$
\tau_{\infty} \leq T
$$

Hence there is an integer $m_{1} \geq m_{0}$ such that

$$
\tau_{m} \leq T, \quad \forall m \geq m_{1} .
$$

Define a $C^{2}$-function $V: R_{+}^{2} \rightarrow \bar{R}_{+}$by

$$
V(x, y)=\left(x-c-c \log \frac{x}{c}\right)+\frac{1}{k}(y-1-\log y),
$$

where $c$ is a positive constant to be determined later. Using the chain rule, we get

$$
\begin{aligned}
\frac{d^{\alpha} V}{d t^{\alpha}} & =\frac{\partial V}{\partial x} \frac{d^{\alpha} x}{d t^{\alpha}}+\frac{\partial V}{\partial y} \frac{d^{\alpha} y}{d t^{\alpha}} \\
& =(x-c)\left(a-b x-\frac{\gamma y}{1+\beta x}\right)
\end{aligned}
$$




$$
\begin{aligned}
& +\frac{1}{k}(y-1)\left(-e+\frac{k \gamma x}{1+\beta x}\right) \\
\leq & -a c+\frac{e}{k}+(a+b c) x-b x^{2}-\left(\frac{e}{k}-\gamma c\right) y .
\end{aligned}
$$

Choose $c=e / k \gamma$ such that $(e / k)-\gamma c=0$; then

$$
\begin{aligned}
\frac{d^{\alpha} V}{d t^{\alpha}} & \leq-a c+\frac{e}{k}+(a+b c) x-b x^{2} \\
& =-b\left(x-\frac{a+b c}{2 b}\right)^{2}+\frac{(a+b c)^{2}}{4 b}+\frac{e}{k}-a c \\
& \leq \frac{(a+b c)^{2}}{4 b}+\frac{e}{k}=K,
\end{aligned}
$$

where $K$ is a positive constant. Then

$$
d^{\alpha} V \leq K d t^{\alpha} \text {. }
$$

Therefore, on the one hand,

$$
\begin{aligned}
\int_{0}^{\tau_{m} \wedge T} d^{\alpha} V(x(t), y(t)) & \leq \int_{0}^{\tau_{m} \wedge T} K d t^{\alpha} \\
& \leq K \int_{0}^{T} d t^{\alpha}
\end{aligned}
$$

Using the equality (13), we have

$$
\int_{0}^{T} d t^{\alpha}=\alpha \int_{0}^{T}(T-t)^{\alpha-1} d t, \quad 0<\alpha \leq 1
$$

which implies that

$$
\begin{aligned}
\int_{0}^{\tau_{m} \wedge T} d^{\alpha} V(x(t), y(t)) & \leq K \alpha \int_{0}^{T}(T-t)^{\alpha-1} d t \\
& =K T^{\alpha} .
\end{aligned}
$$

On the other hand, using the differential relation (9),

$$
\begin{aligned}
\int_{0}^{\tau_{m} \wedge T} & d^{\alpha} V \\
= & \int_{0}^{\tau_{m} \wedge T} \Gamma(1+\alpha) d V=\Gamma(1+\alpha) \\
& \times\left[V\left(x\left(\tau_{m} \wedge T\right), y\left(\tau_{m} \wedge T\right)\right)-V(x(0), y(0))\right] .
\end{aligned}
$$

Therefore,

$$
V\left(x\left(\tau_{m} \wedge T\right), y\left(\tau_{m} \wedge T\right)\right) \leq V(x(0), y(0))+\frac{K T^{\alpha}}{\Gamma(1+\alpha)} .
$$

Set $\Omega_{m}=\left\{\tau_{m} \leq T\right\}$ for $m \geq m_{1}$. Note that there is at least one of $x\left(\tau_{m}\right)$ and $y\left(\tau_{m}\right)$ that equals either $m$ or $1 / m$; then

$$
\begin{aligned}
V( & \left.x\left(\tau_{m}\right), y\left(\tau_{m}\right)\right) \\
\geq & \left(m-c-c \log \frac{m}{c}\right) \wedge\left(\frac{1}{m}-c+c \log (c m)\right) \\
& \wedge \frac{1}{k}(m-1-\log m) \wedge \frac{1}{k}\left(\frac{1}{m}-1+\log m\right) .
\end{aligned}
$$

Hence,

$$
\begin{aligned}
V( & x(0), y(0))+\frac{1}{\Gamma(1+\alpha)} K T^{\alpha} \\
& \geq\left(m-c-c \log \frac{m}{c}\right) \wedge\left(\frac{1}{m}-c+c \log (c m)\right) \\
& \wedge \frac{1}{k}(m-1-\log m) \wedge \frac{1}{k}\left(\frac{1}{m}-1+\log m\right) .
\end{aligned}
$$

Let $m \rightarrow \infty$ lead to the contradiction that $\infty>V(x(0)$, $y(0))+(1 / \Gamma(1+\alpha)) K T^{\alpha}=\infty$. So we must therefore have $\tau_{\infty}=$ $\infty$.

\section{Lyapunov Stability Theory}

Consider the equation

$$
\frac{d^{\alpha} x}{d t^{\alpha}}=f(x)
$$

Here, $x$ and $f(x)$ are $n$-dimensional column vectors. Suppose that $f(0)=0$ and $f(x)$ is continuous in $G:|x|<H$ and satisfies local Lipschitz condition.

Definition 5 (Lyapunov Stability). $x=\varphi\left(t, \tau, \xi_{0}\right)$ is said to be stable (or Lyapunov stable) if, given $\varepsilon>0$, there exists a $\delta>0$ such that, for any $\xi$ satisfying $\left|\xi-\xi_{0}\right|<\delta$, the solution of (28) is defined for $t \geq \tau$ and has the following inequality:

$$
\left|\varphi(t, \tau, \xi)-\varphi\left(t, \tau, \xi_{0}\right)\right|<\varepsilon, \quad t \geq \tau \text {. }
$$

We remark that a solution which is not stable is said to be unstable.

Definition 6 (asymptotic stability). $x=\varphi\left(t, \tau, \xi_{0}\right)$ is said to be asymptotically stable if it is Lyapunov stable and there exists $\delta_{0}>0$ such that, for any $\xi$ satisfying $\left|\xi-\xi_{0}\right| \leq \delta_{0}$, $\lim _{t \rightarrow \infty}\left(\varphi(t, \tau, \xi)-\varphi\left(t, \tau, \xi_{0}\right)\right)=0$.

Define a $C^{1}$-scalar function $V(x)$ in

$$
|x| \leq h<H
$$

Using the chain rule, we have

$$
\frac{d^{\alpha} V}{d t^{\alpha}}=\sum_{i=1}^{n} \frac{\partial V}{\partial x_{i}} \frac{d^{\alpha} x_{i}}{d t^{\alpha}}=\sum_{i=1}^{n} \frac{\partial V}{\partial x_{i}} f_{i}(x)
$$

Theorem 7. Let $V(x)$ be $C^{1}$ function satisfying $V(0)=0$, $V(x)>0$.

(i) If $d^{\alpha} V / d t^{\alpha} \leq 0$, then system (28) has a stable null solution;

(ii) if $d^{\alpha} V / d t^{\alpha}<0$, then system (28) has an asymptotically stable null solution;

(iii) the null solution of system (28) is unstable provided that $d^{\alpha} V / d t^{\alpha}>0$. 
Proof. Consider the following.

Case $1\left(\left(d^{\alpha} V / d t^{\alpha}\right) \leq 0\right)$. Consider

$\forall \varepsilon>0 \quad(\varepsilon \leq h), \quad$ let $r_{\varepsilon}=\min _{\varepsilon \leq|x| \leq h} V(x), \quad r_{\varepsilon}>0$.

Obviously,

$$
\begin{array}{r}
\exists \delta=\delta(\varepsilon)>0, \quad \text { s.t. }|V(x)-V(0)|=V(x)<\varepsilon, \\
\text { for }|x|<\delta .
\end{array}
$$

Let $\varepsilon=r_{\varepsilon}$; then $V(x)<r_{\varepsilon}$.

Let $|\xi|<\delta(\varepsilon)$; the right maximal interval of existence of $x=\varphi(t, \tau, \xi)$ for (30) is $\tau \leq t<t_{1}$.

That is to say, for $\tau \leq t<t_{1}$,

$$
\frac{d^{\alpha} V}{d t^{\alpha}}(\varphi(t, \tau, \xi))=\sum_{i=1}^{n} \frac{\partial V}{\partial x_{i}}(\varphi(t, \tau, \xi)) \cdot f_{i}(\varphi(t, \tau, \xi)) \leq 0 .
$$

Then $d^{\alpha} V \leq 0$.

Using (9),

$$
\frac{d^{\alpha} V}{d t^{\alpha}}=\Gamma(1+\alpha) \frac{d V}{d t} \leq 0
$$

Therefore,

$$
V(\varphi(t, \tau, \xi)) \leq V(\xi)<r_{\varepsilon}, \quad \tau \leq t<t_{1} .
$$

Thus, from (32), we obtain

$$
|\varphi(t, \tau, \xi)|<\varepsilon, \quad \tau \leq t<t_{1} .
$$

Case $2\left(\left(d^{\alpha} V / d t^{\alpha}\right)<0\right)$. We can easily know that the system (28) has a stable null solution. We will consider that there exists $\delta_{0}>0$ such that for any $\xi$ satisfying $|\xi|<\delta_{0}$ then $\lim _{t \rightarrow+\infty} \varphi(t, \tau, \xi)=0$.

Let $\delta_{0}>0$; then $|\varphi(t, \tau, \xi)|<\varepsilon \leq h$ for $|\xi|<\delta_{0}, t \geq \tau$. $\varphi(t, \tau, \xi)$ is bounded for $t \geq \tau$; then there exists increasing $t_{k}$ which converges to $+\infty$ such that

$$
\lim _{k \rightarrow+\infty} \varphi\left(t_{k}, \tau, \xi\right)=\bar{\xi}
$$

Suppose $\bar{\xi} \neq 0$. One obtains

$$
\varphi(t, \tau, \xi) \neq 0, \quad t \geq \tau .
$$

Since $d^{\alpha} V / d t^{\alpha}<0$, then

$$
\frac{d^{\alpha} V(\varphi(t, \tau, \bar{\xi}))}{d t^{\alpha}}<0, \quad t \geq \tau .
$$

Using (9) yields

$$
\frac{d^{\alpha} V(\varphi(t, \tau, \bar{\xi}))}{d t^{\alpha}}=\Gamma(1+\alpha) \frac{d V(\varphi(t, \tau, \bar{\xi}))}{d t}<0 .
$$

Therefore, we have

$$
V(\varphi(t, \tau, \bar{\xi}))<V(\bar{\xi}), \quad t>\tau .
$$

Besides, for any $t$ satisfying $t>\tau$,

$$
\frac{d^{\alpha} V}{d t^{\alpha}}<0
$$

which implies that

$$
V\left(\varphi\left(t_{k}, \tau, \xi\right)\right)<V(\varphi(t, \tau, \xi)), \quad t_{k}>t .
$$

Then according to (38),

$$
\lim _{k \rightarrow \infty} V\left(\varphi\left(t_{k}, \tau, \xi\right)\right)=V(\bar{\xi})<V(\varphi(t, \tau, \xi)), \quad t>\tau .
$$

It is easy to verify that, for any $k$,

$$
\varphi\left(t+t_{k}, \tau, \xi\right)=\varphi\left(t+\tau, \tau, \varphi\left(t_{k}, \tau, \xi\right)\right) .
$$

Note that

$$
\varphi\left(1+t_{k}, \tau, \xi\right)=\varphi\left(1+\tau, \tau, \varphi\left(t_{k}, \tau, \xi\right)\right),
$$

when $t=1$, which implies that

$$
V\left(\varphi\left(1+t_{k}, \tau, \xi\right)\right)=V\left(\varphi\left(1+\tau, \tau, \varphi\left(t_{k}, \tau, \xi\right)\right)\right) .
$$

Let $k \rightarrow+\infty$ and note that $V(x)$ is continuous and $\varphi(t, \tau, \xi)$ is continuous for initial value; we have

$$
V\left(\varphi\left(1+t_{k}, \tau, \xi\right)\right)=V\left(\varphi\left(1+\tau, \tau, \varphi\left(t_{k}, \tau, \xi\right)\right)\right)<V(\bar{\xi}),
$$

which contradicts (45).

Thus, $\bar{\xi}=0$.

Case $3\left(\left(d^{\alpha} V / d t^{\alpha}\right)>0\right)$. Suppose system (28) has a stable null solution; then $\forall \varepsilon>0, \exists \delta(\varepsilon)>0,|\xi|<\delta(\varepsilon)$; we have $|\varphi(t, \tau, \xi)|<\varepsilon, t \geq \tau$.

Let $\xi \neq 0,|\xi|<\delta(\varepsilon)$; according to condition (iii) in Theorem 7 , we know

$$
V(\varphi(t, \tau, \xi))>V(\xi)>0, \quad t>\tau .
$$

Then there exists $\alpha>0$ satisfying $|\varphi(t, \tau, \xi)| \geq \alpha$.

Next, we show $d^{\alpha} V(\varphi(t, \tau, \xi)) / d t^{\alpha}>0$; then there exists $M>0$, such that

$$
\frac{d^{\alpha} V(\varphi(t, \tau, \xi))}{d t^{\alpha}} \geq M
$$

Thus,

$$
\begin{aligned}
V & (\varphi(t, \tau, \xi)) \\
& >V(\varphi(t, \tau, \xi))-V(\varphi(\tau, \tau, \xi)) \\
& =V(\varphi(t, \tau, \xi))-V(\xi) \\
& >\frac{M}{\Gamma(1+\alpha)}(t-\tau)^{\alpha}, \quad t \geq \tau .
\end{aligned}
$$

Noting that $V(\varphi(t, \tau, \xi))$ is bounded and $(M / \Gamma(1+\alpha))(t-\tau)^{\alpha}$ is unbounded when $t \geq \tau$, this contradiction shows that the null solution of system (28) is unstable when $d^{\alpha} V / d t^{\alpha}>0$. 
Example 8. Consider the stability of the null solution of system:

$$
\frac{d^{\alpha} x}{d t^{\alpha}}=y^{2 p-1}, \quad \frac{d^{\alpha} y}{d t^{\alpha}}=-x^{2 p-1},
$$

where $p \in N$. Define a function $V(x, y)=\left(x^{2 p} / 2 p\right)+$ $\left(y^{2 p} / 2 p\right)$. Then $V(0,0)=0, V(x, y)>0,(x, y) \neq(0,0)$, $d^{\alpha} V / d t^{\alpha}=0$. Here by Theorem 7 the system has a stable solution.

Example 9. Consider the stability of the null solution of system:

$$
\begin{aligned}
& \frac{d^{\alpha} x}{d t^{\alpha}}=2 y+y z-x^{3}, \\
& \frac{d^{\alpha} y}{d t^{\alpha}}=-x-x z-y^{3}, \\
& \frac{d^{\alpha} z}{d t^{\alpha}}=x y-z^{3} .
\end{aligned}
$$

Define a function $V(x, y, z)=p x^{2}+q y^{2}+r z^{2}$, where $p, q, r>$ 0 . Obviously, $V(0,0,0)=0, V(x, y, z)>0,(x, y, z) \neq(0,0,0)$, $d^{\alpha} V / d t^{\alpha}=2(2 p-q) x y+2(p-q+r) x y z-2\left(p x^{4}+q y^{4}+r z^{4}\right)$; we can choose the constants $p, q, r$ such that $(2 p-q)=0$, $p-q+r=0$. Then $d^{\alpha} V / d t^{\alpha}<0$ and by Theorem 7 the system has an asymptotically stable null solution.

\section{The Stability of the Solution}

Since $a k \gamma>a e \beta+b e$, then there is a positive equilibrium $\left(x^{*}, y^{*}\right)$ of system (1), and

$$
a=b x^{*}+\frac{\gamma y^{*}}{1+\beta x^{*}}, \quad e=\frac{k \gamma x^{*}}{1+\beta x^{*}} .
$$

Let

$$
\begin{aligned}
V_{1}(x, y)= & \left(x-x^{*}-x^{*} \log \frac{x}{x^{*}}\right) \\
& +a_{1}\left(y-y^{*}-y^{*} \log \frac{y}{y^{*}}\right),
\end{aligned}
$$

where $a_{1}$ is a positive constant to be determined later. Then using the chain rule, we have

$$
\begin{aligned}
\frac{d^{\alpha} V_{1}}{d t^{\alpha}}= & \frac{\partial V_{1}}{\partial x} \frac{d^{\alpha} x}{d t^{\alpha}}+\frac{\partial V_{1}}{\partial y} \frac{d^{\alpha} y}{d t^{\alpha}}=\left(x-x^{*}\right)\left(a-b x-\frac{\gamma y}{1+\beta x}\right) \\
& +a_{1}\left(y-y^{*}\right)\left(-e+\frac{k \gamma x}{1+\beta x}\right) \\
= & -b\left(x-x^{*}\right)^{2}+\frac{\gamma \beta y^{*}}{\left(1+\beta x^{*}\right)(1+\beta x)}\left(x-x^{*}\right)^{2} \\
& -\frac{\gamma}{1+\beta x}\left(x-x^{*}\right)\left(y-y^{*}\right) \\
& +\frac{a_{1} k \gamma}{\left(1+\beta x^{*}\right)(1+\beta x)}\left(x-x^{*}\right)\left(y-y^{*}\right)
\end{aligned}
$$

$$
\begin{aligned}
\leq & -\left(b-\frac{\gamma \beta y^{*}}{1+\beta x^{*}}\right)\left(x-x^{*}\right)^{2} \\
& -\frac{\gamma}{1+\beta x}\left(1-\frac{a_{1} k}{1+\beta x^{*}}\right)\left(x-x^{*}\right)\left(y-y^{*}\right) .
\end{aligned}
$$

Choose $a_{1}=\left(1+\beta x^{*}\right) / k$ such that $1-\left(a_{1} k /\left(1+\beta x^{*}\right)\right)=0$ yields

$$
\frac{d^{\alpha} V_{1}}{d t^{\alpha}} \leq-\left(b-\frac{\gamma \beta y^{*}}{1+\beta x^{*}}\right)\left(x-x^{*}\right)^{2} .
$$

Let

$$
\begin{aligned}
& V_{2}(x, y)=\frac{1}{2}\left[\left(x-x^{*}\right)+\frac{1}{k}\left(y-y^{*}\right)\right]^{2}, \\
& \widetilde{V_{2}}(x, y)=\left(x-x^{*}\right)+\frac{1}{k}\left(y-y^{*}\right) .
\end{aligned}
$$

Note that

$$
\begin{aligned}
\frac{d^{\alpha} \widetilde{V_{2}}}{d t^{\alpha}} & =\frac{\partial \widetilde{V_{2}}}{\partial x} \frac{d^{\alpha} x}{d t^{\alpha}}+\frac{\partial \widetilde{V_{2}}}{\partial y} \frac{d^{\alpha} y}{d t^{\alpha}} \\
& =x\left(a-b x-\frac{\gamma y}{1+\beta x}\right)+\frac{y}{k}\left(-e+\frac{k \gamma x}{1+\beta x}\right) \\
& =-b x\left(x-x^{*}\right)+\gamma \frac{y^{*}\left(x-x^{*}\right)-x^{*}\left(y-y^{*}\right)}{1+\beta x^{*}} .
\end{aligned}
$$

Then

$$
\begin{aligned}
\frac{d^{\alpha} V_{2}}{d t^{\alpha}}= & {\left[\left(x-x^{*}\right)+\frac{1}{k}\left(y-y^{*}\right)\right] \frac{d^{\alpha} \widetilde{V_{2}}}{d t^{\alpha}} } \\
= & {\left[\left(x-x^{*}\right)+\frac{1}{k}\left(y-y^{*}\right)\right] } \\
& \times\left[-b x\left(x-x^{*}\right)+\gamma \frac{y^{*}\left(x-x^{*}\right)-x^{*}\left(y-y^{*}\right)}{1+\beta x^{*}}\right] \\
= & -b x\left(x-x^{*}\right)^{2}+\left(\frac{\gamma y^{*}}{1+\beta x^{*}}+\frac{b y^{*}}{k}\right)\left(x-x^{*}\right)^{2} \\
& -\frac{b}{k}\left(x-x^{*}\right)^{2} y-\frac{\gamma x^{*}}{k\left(1+\beta x^{*}\right)}\left(y-y^{*}\right)^{2} \\
& +\left[\frac{\gamma y^{*}}{k\left(1+\beta x^{*}\right)}-\frac{\gamma x^{*}}{1+\beta x^{*}}-\frac{b x^{*}}{k}\right] \\
& \times\left(x-x^{*}\right)\left(y-y^{*}\right) \\
\leq & \left(\frac{\gamma y^{*}}{1+\beta x^{*}}+\frac{b y^{*}}{k}\right)\left(x-x^{*}\right)^{2}-\frac{\gamma x^{*}}{k\left(1+\beta x^{*}\right)}\left(y-y^{*}\right)^{2} \\
& +\left[\frac{\gamma y^{*}}{k\left(1+\beta x^{*}\right)}-\frac{\gamma x^{*}}{1+\beta x^{*}}-\frac{b x^{*}}{k}\right] \\
& \times\left(x-x^{*}\right)\left(y-y^{*}\right) .
\end{aligned}
$$


Note that

$$
\begin{aligned}
& {\left[\frac{\gamma y^{*}}{k\left(1+\beta x^{*}\right)}-\frac{\gamma x^{*}}{1+\beta x^{*}}-\frac{b x^{*}}{k}\right]\left(x-x^{*}\right)\left(y-y^{*}\right)} \\
& \leq \frac{\left[\left(\gamma y^{*} / k\left(1+\beta x^{*}\right)\right)-\left(\gamma x^{*} /\left(1+\beta x^{*}\right)\right)-\left(b x^{*} / k\right)\right]^{2}}{2\left(\gamma x^{*} / k\left(1+\beta x^{*}\right)\right)} \\
& \quad \times\left(x-x^{*}\right)^{2}+\frac{1}{2} \frac{\gamma x^{*}}{k\left(1+\beta x^{*}\right)}\left(y-y^{*}\right)^{2} \\
& :=\delta\left(x-x^{*}\right)^{2}+\frac{1}{2} \frac{\gamma x^{*}}{k\left(1+\beta x^{*}\right)}\left(y-y^{*}\right)^{2} .
\end{aligned}
$$

Then

$$
\begin{aligned}
\frac{d^{\alpha} V_{2}}{d t^{\alpha}} \leq & \left(\frac{\gamma y^{*}}{1+\beta x^{*}}+\frac{b y^{*}}{k}+\delta\right)\left(x-x^{*}\right)^{2} \\
& -\frac{1}{2} \frac{\gamma x^{*}}{k\left(1+\beta x^{*}\right)}\left(y-y^{*}\right)^{2} .
\end{aligned}
$$

Consider a Lyapunov function $V(x, y)$ defined by

$$
V(x, y)=V_{1}(x, y)+a_{2} V_{2}(x, y)
$$

where $a_{2}$ is a positive constant to be determined later. Then

$$
\begin{aligned}
\frac{d^{\alpha} V}{d t^{\alpha}}= & \frac{d^{\alpha} V_{1}}{d t^{\alpha}}+a_{2} \frac{d^{\alpha} V_{2}}{d t^{\alpha}} \leq-\left(b-\frac{\gamma \beta y^{*}}{1+\beta x^{*}}\right)\left(x-x^{*}\right)^{2} \\
& +a_{2}\left(\frac{\gamma y^{*}}{1+\beta x^{*}}+\frac{b y^{*}}{k}+\delta\right)\left(x-x^{*}\right)^{2} \\
& -\frac{a_{2}}{2} \frac{\gamma x^{*}}{k\left(1+\beta x^{*}\right)}\left(y-y^{*}\right)^{2} \\
= & -\left[b-\frac{\gamma \beta y^{*}}{1+\beta x^{*}}-a_{2}\left(\frac{\gamma y^{*}}{1+\beta x^{*}}+\frac{b y^{*}}{k}+\delta\right)\right] \\
& \times\left(x-x^{*}\right)^{2}-\frac{a_{2}}{2} \frac{\gamma x^{*}}{k\left(1+\beta x^{*}\right)}\left(y-y^{*}\right)^{2} .
\end{aligned}
$$

Choose $a_{2}>0$ such that

$$
\begin{gathered}
b-\frac{\gamma \beta y^{*}}{1+\beta x^{*}}-a_{2}\left(\frac{\gamma y^{*}}{1+\beta x^{*}}+\frac{b y^{*}}{k}+\delta\right) \\
=\frac{1}{2}\left(b-\frac{\gamma \beta y^{*}}{1+\beta x^{*}}\right) .
\end{gathered}
$$

Then it follows from (65) that

$$
\begin{aligned}
\frac{d^{\alpha} V}{d t^{\alpha}} \leq & -\frac{1}{2}\left(b-\frac{\gamma \beta y^{*}}{1+\beta x^{*}}\right)\left(x-x^{*}\right)^{2} \\
& -\frac{a_{2}}{2} \frac{\gamma x^{*}}{k\left(1+\beta x^{*}\right)}\left(y-y^{*}\right)^{2}
\end{aligned}
$$

According to (66),

$$
b-\frac{\gamma \beta y^{*}}{1+\beta x^{*}}=2 a_{2}\left(\frac{\gamma y^{*}}{1+\beta x^{*}}+\frac{b y^{*}}{k}+\delta\right)>0 .
$$

Thus,

$$
\frac{d^{\alpha} V}{d t^{\alpha}}<0
$$

Hence, from these arguments, we get the following result.

Theorem 10. If $a k \gamma>a e \beta+$ be such that system (1) has a positive equilibrium $\left(x^{*}, y^{*}\right)$ and $a_{2}$ is defined as in the proof, then system (1) is asymptotically stable.

\section{Conflict of Interests}

The author declares that there is no conflict of interests regarding the publication of this paper.

\section{Acknowledgment}

The author thanks Professor Y. Li for his valuable discussion.

\section{References}

[1] H. I. Freedman, Deterministic Mathematical Models in Population Ecology, vol. 57 of Monographsand Textbooks in Pure and Applied Mathematics, Marcel Dekker, New York, NY, USA, 1980.

[2] C. S. Holling, "The components of predation as revealed by a study of small-mammal predation of the european pine sawy," Canadian Entomologist, vol. 91, no. 5, pp. 293-320, 1959.

[3] Z. W. Liu, N. Z. Shi, D. Q. Jiang, and C. Y. Ji, “The asymptotic behavior of a stochastic predator-prey system with Holling II functional response," Abstract and Applied Analysis, vol. 2012, Article ID 801812, 14 pages, 2012.

[4] L. S. Chen and Z. J. Jing, "Existence and uniqueness of limit cycles for the differential equations of predator-prey interaction," Kexue Tongbao, vol. 29, no. 9, pp. 521-523, 1984 (Chinese).

[5] X. N. Liu and L. S. Chen, "Complex dynamics of Holling type II Lotka-Volterra predator-prey system with impulsive perturbations on the predator," Chaos, Solitons and Fractals, vol. 16, no. 2, pp. 311-320, 2003.

[6] S. W. Zhang and L. S. Chen, "A Holling II functional response food chain model with impulsive perturbations," Chaos, Solitons and Fractals, vol. 24, no. 5, pp. 1269-1278, 2005.

[7] L. N. Song and W. G. Wang, "Solution of the fractional BlackScholes option pricing model by finite difference method," Abstract and Applied Analysis, vol. 2013, Article ID 194286, 10 pages, 2013.

[8] A. Atangana and A. Secer, "The time-fractional coupledKorteweg-de-Vries equations," Abstract and Applied Analysis, vol. 2013, Article ID 947986, 8 pages, 2013.

[9] I. Karatay and S. R. Bayramoglu, "A characteristic difference scheme for time-fractional heat equations based on the CrankNicholson difference schemes," Abstract and Applied Analysis, vol. 2012, Article ID 548292, 11 pages, 2012.

[10] A. Golbabai and K. Sayevand, "Solitary pattern solutions for fractional Zakharov-Kuznetsov equations with fully nonlinear dispersion," Applied Mathematics Letters, vol. 25, no. 4, pp. 757766, 2012. 
[11] H. Jafari, H. Tajadodi, N. Kadkhoda, and D. Baleanu, "Fractional subequation method for Cahn-Hilliard and KleinGordon equations," Abstract and Applied Analysis, vol. 2013, Article ID 587179, 5 pages, 2013.

[12] G. Jumarie, "Modified Riemann-Liouville derivative and fractional Taylor series of nondifferentiable functions further results," Computers \& Mathematics with Applications, vol. 51, no. 9-10, pp. 1367-1376, 2006.

[13] G. Jumarie, "The Minkowski's space-time is consistent with differential geometry of fractional order," Physics Letters A, vol. 363, no. 1-2, pp. 5-11, 2007.

[14] A. A. Kilbas, H. M. Srivastava, and J. J. Trujillo, Theory and Applications of Fractional Differential Equations, vol. 204, Elsevier Science B.V., Amsterdam, The Netherlands, 2006.

[15] K. S. Miller and B. Ross, An Introduction to the Fractional Calculus and Fractional Differential Equations, John Wiley \& Sons, New York, NY, USA, 1993.

[16] I. Podlubny, Fractional Differential Equations, vol. 198, Academic Press, San Diego, Calif, USA, 1999.

[17] S. G. Samko, A. A. Kilbas, and O. I. Marichev, Fractional Integrals and Derivatives, Translated from the 1987 Russian Original, Gordon and Breach, Yverdon, Switzerland, 1993.

[18] G. Jumarie, "Fractional Hamilton-Jacobi equation for the optimal control of nonrandom fractional dynamics with fractional cost function," Journal of Applied Mathematics \& Computing, vol. 23, no. 1-2, pp. 215-228, 2007.

[19] G. Jumarie, "Table of some basic fractional calculus formulae derived from a modified Riemann-Liouville derivative for nondifferentiable functions," Applied Mathematics Letters, vol. 22, no. 3, pp. 378-385, 2009. 


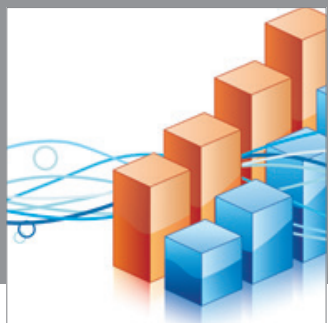

Advances in

Operations Research

mansans

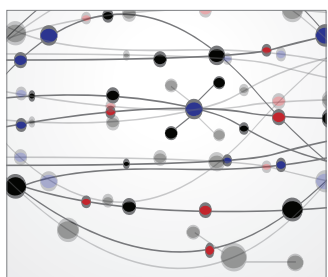

The Scientific World Journal
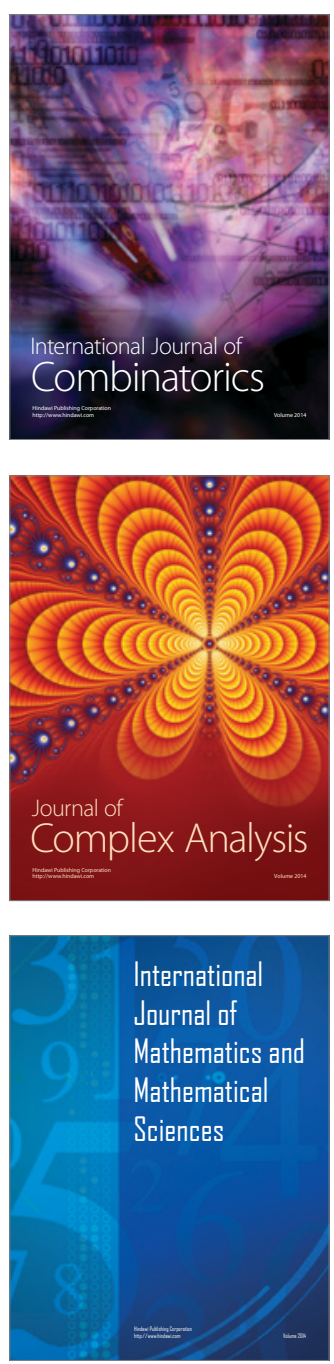
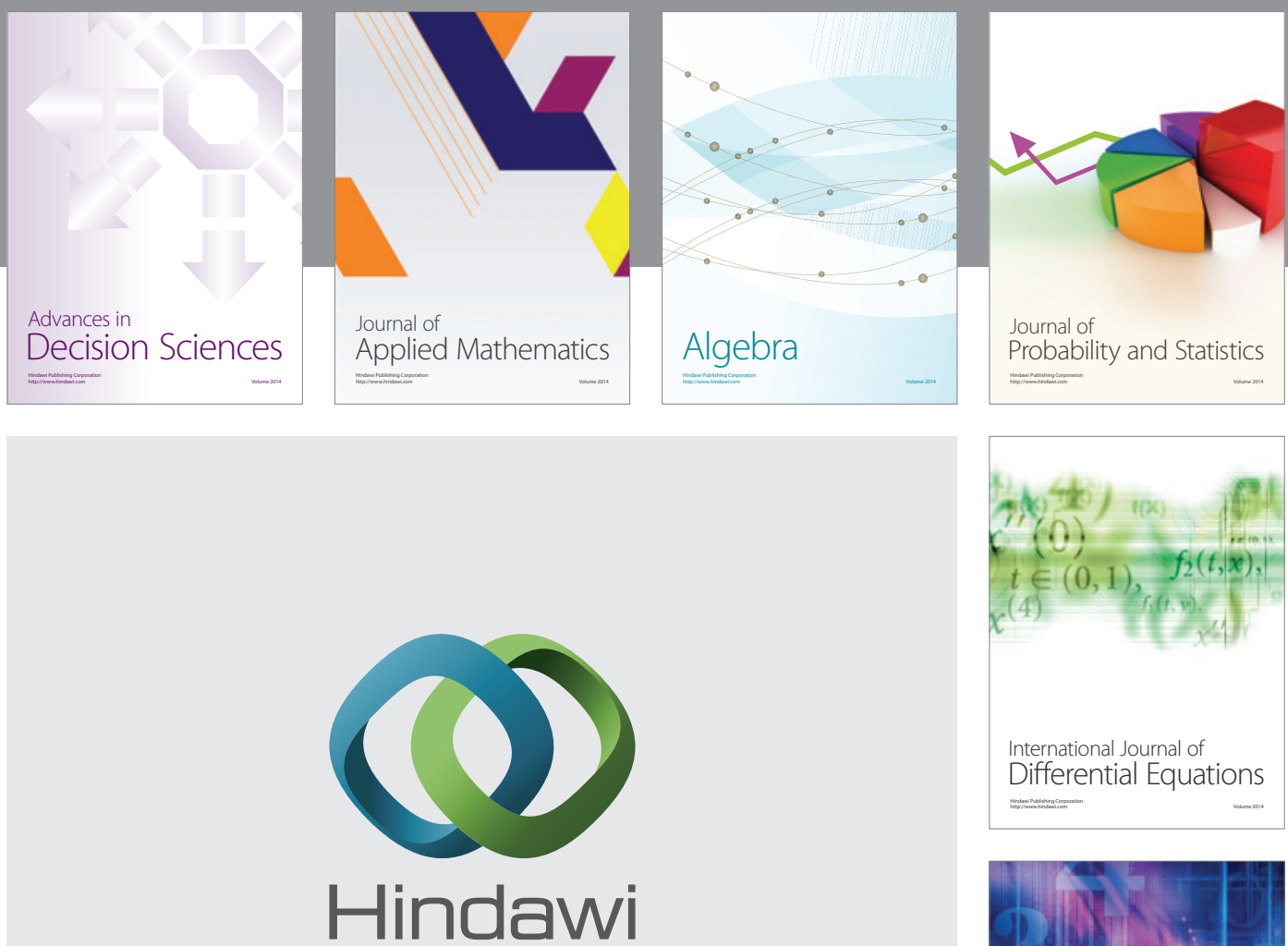

Submit your manuscripts at http://www.hindawi.com
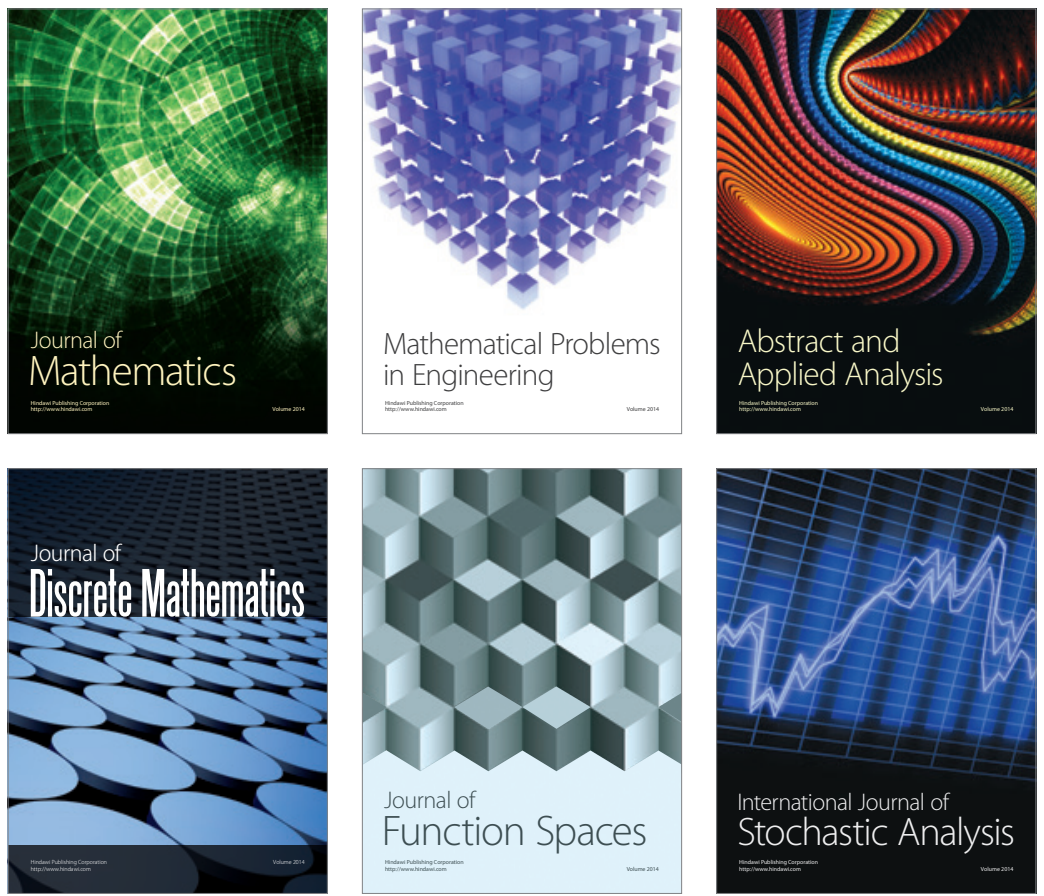

Journal of

Function Spaces

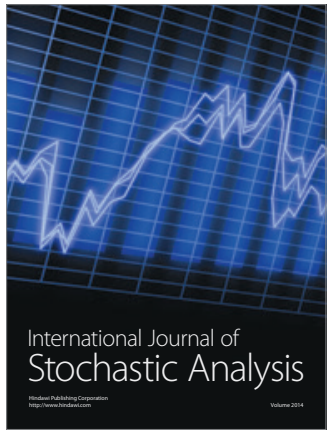

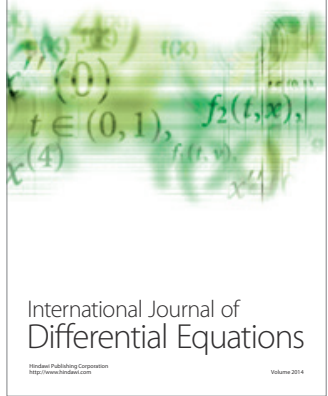
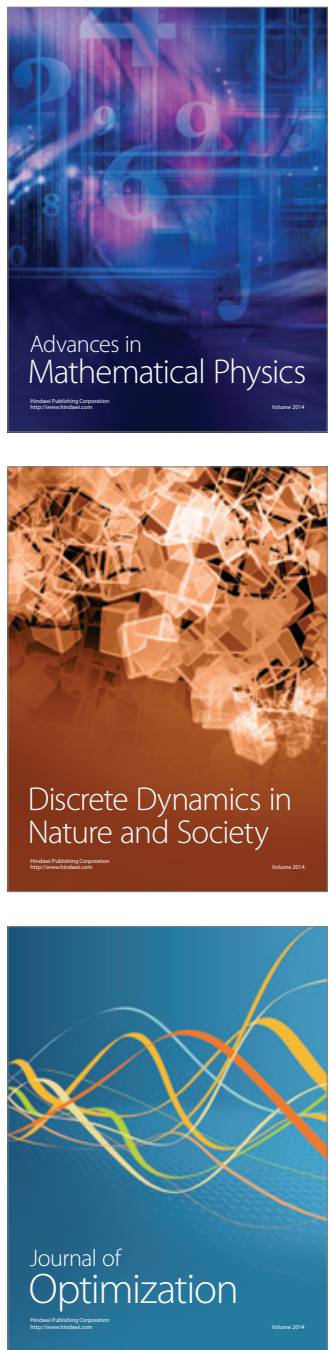